\title{
Iranian Journal of
}

Educational

Sociology

\section{Iranian journal of educational Sociology}

(Interdisciplinary Journal of Education)

Available online at: http://www.iase-idje.ir/

Volume 2, Number 2, July 2019

\section{Comparison of the Efficacy of Transcranial Magnetic Stimulation and Cognitive-Behavioral Therapy on Depression}

\author{
Alireza Salemi Khameneh ${ }^{1}$, Saeed Bakhtiarpour ${ }^{2 *}$, Alireza Heydarei ${ }^{3}$, Farah Naderi ${ }^{4}$, Parvin Ehtesham Zadeh ${ }^{5}$ \\ 1. Ph.D. student of psychology, Department of psychology, khuzestan science and research Branch, Islamic Azad \\ University, Ahvaz, Iran and Ph.D. student of psychology, Department of psychology, Ahvaz Branch, Islamic Azad \\ University, Ahvaz, Iran. \\ 2. Assistant professor of psychology, Department of psychology, Ahvaz Branch, Islamic Azad University, Ahvaz, Iran \\ 3. Assistant professor of psychology, Department of psychology, Ahvaz Branch, Islamic Azad University, Ahvaz, Iran \\ 4. Assistant professor of psychology, Department of psychology, Khuzestan science and research Branch, Islamic Azad \\ University, Ahvaz, Iran and Assistant professor of psychology, Department of psychology, Ahvaz Branch, Islamic Azad \\ University, Ahvaz, Iran. \\ 5. Assistant professor of psychology, Department of psychology, Ahvaz Branch, Islamic Azad University, Ahvaz, Iran.
}

\section{Article history:}

Received date: 11 January 2019

Review date: 26 March 2019

Accepted date: 29 March 2019

\section{Abstract}

Purpose: The aim of this study was to compare the effectiveness of transcranial magnetic stimulation (tms) and cognitivebehavioral therapy (cbt) in patients with depression in Tehran.

Methodology: The purpose of the present study is to apply the pre-test and post-test with control group in terms of semiexperimental research method. The study sample consisted of 60 clients with depression who had been diagnosed by the Psychiatric Commission and admitted to psychology clinics in 2015. They were selected by random sampling and were randomly divided into three groups (two experimental and one control group) in each group of 20 persons. Experimental groups in one group underwent cognitive-behavioral therapy for 12 sessions and the other group underwent transcranial magnetic stimulation for 20 sessions. Control group received no intervention. Data were analyzed using covariance analysis.

Findings: The results showed that there was a significant difference between transcranial magnetic stimulation and cognitive-behavioral therapy in reducing depression in clients $(\mathrm{P}$ $<0.01)$.

Conclusion: Transcranial magnetic stimulation therapy and cognitive-behavioral therapy are effective in reducing depression in patients referred to psychiatric clinics.

\footnotetext{
Please cite this article: Salemi Khamene A, Bakhtiari Pour S, Naderi F, Heidari A, Ehteshamzadeh P. (2019). Comparison of the efficacy of transcranial magnetic stimulation and cognitive-behavioral therapy on depression, Iranian journal of educational Sociology. 2(2):182-187.
}

\footnotetext{
* Corresponding Author Email:Saeedb81@yahoo.com
} 


\section{Introduction}

Depression will be one of the most common and costly diseases predicted by 2020. (Ginzburg \& Solomon, 2010). Depression is a common name for disorders such as disordered mood disorder, major depressive disorder (including major depression), persistent depressive disorder (depression), and depression due to other physical illness (Kessler, et al, 2003). Depression is a mood disorder characterized by feelings of inadequacy, hopelessness, decline in activity or reactivity, pessimism, sadness, and related symptoms (DSM-5, 2013, translated by Seyed Mohammadi, 2014).

Depression Disorders According to the World Health Organization Report Listed by this organization. According to a 2006 report, about $25 \%$ of people in health centers worldwide suffer from these disorders. The report estimates that at any given point in time, 15 to 20 percent of adults suffer from significant levels of depressive symptoms and at least 12 percent have depression that can cure them at some stage in their lives (Lambert, 2006). Psychotherapists use a variety of therapies to treat depression, including transcranial magnetic stimulation (tms) and cognitive-behavioral therapy (cbt). Transcranial magnetic stimulation therapy (tms) is a non-invasive, safe, and painless method that directly stimulates the brain and is nowadays considered one of the most widely used methods in the treatment of mental disorders (George, Wassermann \& Post, 1999). In TMS, a strong current flows through the coil that is placed on the person's head. This electric current generates a local magnetic field of 1.5 to 2 Tesla that passes through the skull, and the magnetic field in turn divides the brain cells to a depth of $2 \mathrm{~cm}$ (depolarizing) (George, et al, 2006).

Evidence from brain imaging has shown that the left hemisphere has a low activity in depression of the dorsolateral prefrontal cortex. Accordingly, repetitive left cerebral prefrontal cortex stimulation (DLPFC) can be stimulated through repetitive cranial magnetic stimulation (TMS) treatment (George, et al, 2000). On the other hand, other research evidence suggests that increased activity of the right hemisphere in the dorsolateral prefrontal cortex can be mitigated by the inhibitory effects induced by low frequency TMS (Klein, 1999). The results of meta-analyzes of the effect of this method on depression have shown that the high frequency stimulation in the left hemisphere (DLPFC) of the experimental group was significantly higher than the virtual group (Martin, et al, 2003). Other evidence has also shown that in depressed patients who are resistant to drug therapy, improved mood persists more than three months after stimulation (Khamami, 2008).

In the last four decades, more than 200 comparative study articles on The efficacy of psychological therapies has been compared to other therapies. These studies have shown that psychological therapy has a significant effect on depression in adults. And cognitive-behavioral therapy has shown the most evidence of therapeutic efficacy. Notable is the success of standard cognitive-behavioral therapy in the treatment of depression, as well as in the reduction of recurrence rates compared to other treatments. Cognitivebehavioral therapy took place in 3 stages. First, therapeutic behavior emerged independently and in parallel in the United Kingdom and the United States during the 1970s and 1970s. Secondly, cognitive therapy began in the United States from the mid-1960s onwards, and in the third phase, it developed cognitive and behavioral therapies in the form of cognitive behavioral therapy in the late 1980s (Khamami, 2008). For three decades, the following reasons have led to a significant increase in the trend in cognitive-behavioral therapy: 1. Increasing empirical evidence supporting the effectiveness of therapeutic-behavioral therapy for common psychological problems such as depression and anxiety disorders. 2. The cognitive-behavioral approach is relatively short-lived, making it more economically viable than other alternative therapies. 3. The scope of successful use of the cognitive-behavioral approach is for a wide range of disorders, thus providing a significant and useful opportunity for therapists in the general medical profession to train this approach. 4. Detahoo, William, and Hayes (2003) quoted Mirnasab (2006) as a cognitive-behavioral therapeutic system using a variety of individual therapy techniques. Therefore, the present study is concerned with comparing the efficacy of transcranial magnetic stimulation therapy. tms) and cognitive- 
behavioral therapy (cbt) referrals to psychiatric clinics what changes in the severity of their depressive symptoms as well as their social function and if they change over a follow-up period, will their condition stay coma or impact Are these practices cross-sectional?

\section{Methodology}

The present study was applied in terms of purpose and in terms of data collection and treatment plan as a quasi-experimental study with a control group. Statistical population, sample and sampling method: The study population consisted of all patients with depression in Tehran in autumn 2015. The sample consisted of 60 patients who had inclusion criteria of this study mean being over 20 years old and having sex (male and female) with depression. Diagnosed by the Psychiatric Commission and admitted to the psychology clinics and agreed to participate in the study. Exclusion criteria were under 20 years of age and nonparticipation in the study. 20 people were replaced in each group Clinical depression Ngan Participants by Beck Depression Inventory were measured at pretest-posttest. Experimental group, one group received Experimental Cognitive-Behavioral Therapy for 12 sessions, and Experimental Group two received transcranial magnetic stimulation for 20 sessions. Use in clinics including frequency of $10 \mathrm{~Hz}$ for $5 \mathrm{~s}$ stimulation time, $16 \mathrm{~s}$ interval for each $16 \mathrm{~s}$ stimulation, 120\% motion threshold, stimulation location equal to $\mathrm{f} 3$ in 10-20 system or LDLPFC and 20 min stimulation time and control group received no intervention. did not. The data were analyzed in two parts: descriptive and inferential statistics, mean and standard deviation were used in the descriptive part. Inferential statistics were used to compare and compare the differences between groups in pre-test and post-test by one-way analysis of covariance (ANCOVA). SPSS software version 22 was used for data analysis. The research tools in this study included Beck Depression Inventory and Beck Anxiety Inventory. 1.Beck Depression Inventory (2000): Beck Depression Inventory is one of the most appropriate tools for measuring depression severity (Beck, 2000). Thus, the total score of the questionnaire ranges from zero to 63. Due to the importance of this tool in the diagnosis of clinical intervention, many psychometric studies have been conducted on its psychometric properties. In 1996, Beck et al. obtained a one-week test-retest reliability coefficient of 0.93. The validity of Beck Depression Inventory was also investigated. Mean correlation of Beck Depression Inventory with Hamilton Psychiatric Rating Scale (HRSD), Zong Self-Assessment Scale, MMPI Depression Scale, Multiple Depression Emotional Trait Scale, and SCL-90 more than 0.60. Is. Thanksgiving and Mehriyar research in 1994 noted that its reliability coefficient in Iran was 0.78 (Fathi Ashtiani, 2009).

\section{Findings}

In order to analyze the research data in two descriptive and inferential descriptive sections were separated and analyzed and analyzed. Then, in the second part of the inferential statistics, the parametric test of covariance analysis of one variable was used to investigate the research hypothesis.

Table1. Descriptive indices for control group in two stages of pre-test and post-test $(n 1=n 2=n 3=20)$

\begin{tabular}{ccccccc}
\hline Index & Variable & Number & At least & Maximum & Average & $\begin{array}{c}\text { Standard } \\
\text { deviation }\end{array}$ \\
\hline \multirow{2}{*}{$\begin{array}{c}\text { Depression } \\
\text { disorder }\end{array}$} & pre-exam & 20 & 32 & 47 & $39 / 60$ & $4 / 185$ \\
\cline { 2 - 7 } & Post-test & 20 & 31 & 45 & $38 / 65$ & $4 / 171$ \\
\hline
\end{tabular}

The information in Table 1 is the mean and standard deviation of "Depression Disorder Indicators" in the pre-test and post-test stages for the control group. As it is visible The difference between the scores in the pre-test and post-test stages is small. 
185| Comparison of the efficacy of transcranial magnetic ...Volume 2, Number 2, 2019

Table2. Descriptive indices for experimental group 1 in two stages of pre-test and post-test $(n 1=n 2=n 3=20)$

\begin{tabular}{cccccccc}
\hline Index & Variable & Number & At least & Maximum & Average & Standard deviation \\
\hline \multirow{2}{*}{ Depression disorder } & pre-exam & 20 & 34 & 47 & $39 / 50$ & $3 / 963$ \\
\cline { 2 - 8 } & Post-test & 20 & 15 & 26 & $19 / 15$ & $3 / 233$ \\
\hline
\end{tabular}

Data shown in Table (2) are averages and standard deviations of the "Depression Indicators" in the pretest and post-test stages for the experimental group one. As it is visible Almost in terms of depressive disorder, the difference between scores in the pre-test and post-test stages is high.

Table 3. Descriptive indices for experimental group 2 in two stages of pre-test and post-test $(n 1=n 2=n 3=20)$

\begin{tabular}{ccccccc}
\hline Index & Variable & Number & At least & Maximum & Average & $\begin{array}{c}\text { Standard } \\
\text { deviation }\end{array}$ \\
\hline \multirow{2}{*}{$\begin{array}{c}\text { Depression } \\
\text { disorder }\end{array}$} & pre-exam & 20 & 30 & 50 & $36 / 75$ & $4 / 833$ \\
\cline { 2 - 8 } & Post-test & 20 & 15 & 27 & $19 / 30$ & $3 / 230$ \\
\hline
\end{tabular}

The data shown in Table (3) are the mean and standard deviation of the Depression Indicator Index in the pre-test and post-test for the two experimental groups. As it is visible Almost in the depression disorder index, the difference between scores in the pre-test and post-test stages is high. Covariance analysis was used to test the hypothesis of this study in inferential statistics analysis. Research hypothesis There is a difference between the effectiveness of cognitive behavioral therapy and transcranial magnetic stimulation therapy on depression.

Before performing the covariance analysis, the hypotheses of this method, normal distribution of variables, homogeneity of regression line and homogeneity of variances were used to test the research hypothesis The results of Levin test confirmed the homogeneity of variance of the groups. In order to determine the effectiveness of cognitive behavioral therapy and transcranial magnetic stimulation therapy on reducing depression in participants, a one-way covariance analysis (ANCOVA) was performed. The results are summarized in the following tables.

Table4. Summary of one-way covariance analysis test results

\begin{tabular}{cccccccc}
\hline Variable & $\begin{array}{c}\text { Source of } \\
\text { Changes }\end{array}$ & $\begin{array}{c}\text { Total } \\
\text { Squares }\end{array}$ & $\begin{array}{c}\text { Degrees of } \\
\text { freedom }\end{array}$ & $\begin{array}{c}\text { Average } \\
\text { Squares }\end{array}$ & $\begin{array}{c}\text { Coefficient } \\
\text { F }\end{array}$ & $\begin{array}{c}\text { Surface } \\
\text { Meaningful }\end{array}$ & $\begin{array}{c}\text { Coefficient } \\
\text { Impact (eta) }\end{array}$ \\
\hline \multirow{2}{*}{$\begin{array}{c}\text { Depression } \\
\text { disorder }\end{array}$} & Coproduction & $0 / 530$ & 1 & $0 / 530$ & $0 / 097$ & $0 / 757$ & $0 / 002$ \\
\cline { 2 - 8 } & $\begin{array}{c}\text { Effect of } \\
\text { therapy }\end{array}$ & $68 / 429$ & 2 & $34 / 214$ & $6 / 236$ & $0 / 004$ & $0 / 193$ \\
\cline { 2 - 8 } & Error & $285 / 301$ & 52 & $5 / 487$ & & & \\
\cline { 2 - 8 } & Total & 42926 & 60 & & & & \\
\hline
\end{tabular}

The results of Table 6 show that there is a significant difference between the effectiveness of cognitive behavioral therapy and transcranial magnetic stimulation therapy on the level of depression $(\mathrm{P}<0.01, \mathrm{~F}=$ 6.26) in patients with depression. Therefore, it can be concluded that the efficacy of cognitive behavioral therapy and transcranial magnetic stimulation therapy are effective in reducing depression in patients with depressive disorder.

\section{Discussion}

The purpose of this study was to compare the efficacy of transcranial magnetic stimulation (tms) and cognitive-behavioral therapy (cbt) on reducing depression in clients of psychiatric clinics. As the results of the study showed, transcranial magnetic stimulation (tms) treatment was significantly effective in reducing depression symptoms in depressed patients. Recent research findings corroborate previous findings in the effectiveness of transcranial magnetic stimulation therapy on depression. Since 1985, when Barker 1 was able to demonstrate the application of magnetism in the brain by stimulating the cortex of the motor area (M1), extensive research has been done on the effect of magnetic stimulation on the brain in the field of treatment and research. Until the mid-1990s, a number of studies have confirmed the role of cranial wall 
magnetic stimulation (tms) in the treatment of depression (George, et al, 2006). In fact, the majority of these studies have considered DLPFC stimulation to improve depression syndrome, and several metaanalyzes have shown that stimulation of this region in the high-frequency left hemisphere (greater than 1 $\mathrm{Hz}$ ) has a remarkable effect. (Martin., et al, 2003). Although the majority of studies in this area have investigated the effect of this method on symptoms of depressive disorder, some studies have shown that this method has not had a significant effect on the control group (Khamami, 2008). New magnetic stimulation devices are in progress, and on October 8, 2008, this treatment was approved by the US Food and Drug Administration for the treatment of depression and it should be noted that until its final approval by the agency, research is still underway. Considering the newness of this treatment and also the limited number of peugeot so far. Iran has been conducted in this area, this study sought to evaluate the role of this procedure in the treatment of depression As we indicated at the conclusion tms in relieving symptoms of depression had a significant impact. The mechanism of action of this technique is still unknown, but stimulation of the prefrontal cortical area of the forebrain is due to interactions with other brain areas such as the hippocampus, cortex, anterior cingulate, and amygdala (Paus, et all, 2001). The neurotransmitter chemicals such as sertonin, dopamine, adrenaline act as antidepressants (Khamami, 2008).

Cognitive-behavioral therapy (CBT) was also significantly effective in reducing depression symptoms in depressed patients. Recent research findings confirm previous findings on the effectiveness of cognitivebehavioral therapy for depression. Depression therefore responds to cognitive-behavioral intervention. There are several possible explanations for the significant effectiveness of cognitive-behavioral therapy on patients' depression: Provides an opportunity for the sufferer to break free from the needs and utopian ideals. By changing one's perceptions and modifying one's irrational cues, the three-dimensional triangle of attitudes toward the environment and towards the future changes. As a result of the patient instead of focusing on disabilities, anxieties, and frustration and worry. Finds a new, more flexible perspective than the future. The presence of behavioral strategies is also a step towards activating the patient and helping him to get out of the patient's role and resume life and engage in daily activities. Second, cognitive-behavioral therapy with the information and awareness it gives to the patient encourages him, despite his inability, to strive to increase mood. Cognitive-behavioral therapy teaches patients about illness and early symptoms of depression. Including sleep and coping skills training to help relieve symptoms of depression and symptoms of depression help reduce the risk of relapse. Perhaps one of the most valuable aspects of Cognitive Behavioral Therapy is its ability to reduce cognitive symptoms. In this study, this treatment has been successful in reducing the symptoms of depressive symptoms. It also improves patients' self-esteem because of the patient's familiarity with the nature of the disease and the teaching of non-pharmacological methods to control symptoms. Behavioral control methods are helpful for managing patients' lives after the end of the sessions. Cognitive-behavioral therapy is a patient-centered therapy that helps the patient become involved in the disease process, controlling symptoms and improving themselves. This reduces patients' sense of helplessness when exposed to the disorder. Functional improvement is important because, after undergoing an acute phase of the disease, the patient communicates and re-contacts the social environment around him / her. So it is as important as symptomatic improvement and special attention should be paid to it, eventually that treatment should move from symptomatic improvement to functional improvement. To improve functionality, attention is paid to the subtle symptoms of depressive disorder, and the overall result was that both therapies have significant efficacy, and both are effective in treating depression and support the research hypothesis. 


\section{References}

Beck A. (2000). Manual for the beck depression inventory-II. San Antonio: The psychological corporation.

DSM5 - Diagnostic and Statistical Manual of Mental Disorders 2013, translated by Yahya Seyed Mohammad, 2014.

Fathi Ashtiani A. (2009), Psychological Examinations, Tehran: Bassat Publishing Institute.

George M S, NahasI, Molloy M, Speer A M. (2006). a controlled trial of daily left prefrontal cortex TMS for treating depression. Biological psychiatry; (48):962-78.

George M S, Nahas Z, Molloy M, Speer A M. (2000). A controlled trial of daily eft prefrontal cortex TMS fortreating depression. Neurosci. (8): 373-382.

George M S, Wassermann E M, Post R M. (1999). Transcranial magnetic stimulation a neuro psychiatric tool for the 21 stcentury J.Neuropsychiatry Clin.

Ginzburg K T, Solomon Z. (2010). Comorbidity of posttraumatic Stress, anxiety and depression A 20-yewr Longitudinal study of war veterans.Journal of Affective Disorder,(123): 249-257.

Kessler K, Edelman R, Janeway D, Orlowski B, et all. (2003). The effect of stress management group therapy on patient on an adolescent psychiatric unit. Journal of child and Adolescent Group Therapy, (10): 151- 158.

Khamami S. (2008), Assessment of Social Function and Severity of Depression Symptoms Before and After Repeated Magnetic Resonance Stimulation (rTMS) in Patients with Major Depressive Disorder, MSc Thesis, School of Education and Psychology, University of Tehran.

Klein D N. (1999). Social adjustment in Dysthymia double depression and episodic depression. Affect Discord;(37): 91-101.

Lambert K G. (2006). Rising rates of depression in today society: consideration of roles effort-based rewards and enhanced resilience in day- to- day functioning. Neuroscience and behavioral rewards;(30): 497- 510.

Martin J L, Barbanoj M J, Schlaepfer T E, Thompson E. (2003). Repetitive transcranial magnetic stimulation for the treatment of depression. Systematic review and meta- analysis. British Journal of Psychiatry;(182): 480-491.

Mirnasab, M M. (2006), Effectiveness of Behavioral and Cognitive-Behavioral Therapies in Reducing Clinical Symptoms of Attention Deficit Hyperactivity Disorder, PhD Thesis in Exceptional Child Psychology, University of Tehran.

Paus T, Zatorre R J, Hofle N, Caramanos Z. (2001). Time-related changes in neural systems underlying attention and arousal during the performance of an auditory gilance task Cogn. Neurosci (9): 392-408. 